\title{
On the extreme points of subordination families
}

\author{
by JACEK Dziok (Rzeszów)
}

\begin{abstract}
We investigate extreme points of some classes of analytic functions defined by subordination and classes of functions with varying argument of coefficients. By using extreme point theory we obtain coefficient estimates and distortion theorems in these classes of functions. Some integral mean inequalities are also pointed out.
\end{abstract}

1. Introduction. Let $\widetilde{\mathcal{A}}$ denote the class of functions which are analytic in $\mathcal{U}=\{z \in \mathbb{C}:|z|<1\}$. We consider the usual topology on $\widetilde{\mathcal{A}}$ (see [9]) defined by a metric in which a sequence $\left\{f_{n}\right\}$ in $\widetilde{\mathcal{A}}$ converges to $f$ if and only if it converges to $f$ uniformly on each compact subset of $\mathcal{U}$. It follows from the theorems of Weierstrass and Montel that this topological space is complete.

Let $\mathcal{F}$ be a subclass of $\widetilde{\mathcal{A}}$. A function $f \in \mathcal{F}$ is called an extreme point of $\mathcal{F}$ if the condition

$$
f=\gamma g+(1-\gamma) h \quad(g, h \in \mathcal{F}, 0<\gamma<1)
$$

implies $g=h$. We write $E \mathcal{F}$ for the set of all extreme points of $\mathcal{F}$. It is clear that $E \mathcal{F} \subset \mathcal{F}$.

We say that $\mathcal{F}$ is locally uniformly bounded if for each $r, 0<r<1$, there is a real constant $M=M(r)$ such that

$$
|f(z)| \leq M \quad(f \in \mathcal{F},|z| \leq r) .
$$

We say that a class $\mathcal{F}$ is convex if

$$
\gamma f+(1-\gamma) g \in \mathcal{F} \quad(f, g \in \mathcal{F}, 0 \leq \gamma \leq 1) .
$$

Moreover, we define the closed convex hull of $\mathcal{F}$ as the intersection of all closed convex subsets of $\widetilde{\mathcal{A}}$ that contain $\mathcal{F}$. We denote the closed convex hull of $\mathcal{F}$ by $H \mathcal{F}$.

2010 Mathematics Subject Classification: Primary 30C45; Secondary 30C80.

Key words and phrases: analytic functions, convex functions, extreme points, varying arguments, subordination, Hadamard product. 
If $J: \widetilde{\mathcal{A}} \rightarrow \widetilde{\mathcal{A}}$ is a linear homeomorphism which maps $\mathcal{F}$ onto $J(\mathcal{F})$, then it is easy to verify that

$$
H J(\mathcal{F})=J(H \mathcal{F})
$$

Likewise, if the class

$$
\mathcal{F}=\left\{f_{n} \in \widetilde{\mathcal{A}}: n \in \mathbb{N}=\{1,2, \ldots,\}\right\}
$$

is locally uniformly bounded, then

$$
H \mathcal{F}=\left\{\sum_{n=1}^{\infty} \gamma_{n} f_{n}: \sum_{n=1}^{\infty} \gamma_{n}=1, \gamma_{n} \geq 0(n \in \mathbb{N}\} .\right.
$$

A functional $\mathcal{J}: \widetilde{\mathcal{A}} \rightarrow \mathbb{R}$ is called convex on a convex class $\mathcal{F} \subset \widetilde{\mathcal{A}}$ if

$$
\mathcal{J}(\gamma f+(1-\gamma) g) \leq \gamma \mathcal{J}(f)+(1-\gamma) \mathcal{J}(g) \quad(f, g \in \mathcal{F}, 0 \leq \gamma \leq 1) .
$$

For each fixed $m, n \in \mathbb{N}_{2} z \in \mathcal{U}$ the following real-valued functionals are continuous and convex on $\widetilde{\mathcal{A}}$ :

$$
\mathcal{J}(f)=\left|a_{n}\right|, \quad \mathcal{J}(f)=|f(z)|, \quad \mathcal{J}(f)=\left|f^{(m)}(z)\right| \quad(f \in \widetilde{\mathcal{A}}) .
$$

Moreover, for $\lambda>0,0<r<1$, the real-valued functional

$$
\mathcal{J}(f)=\left(\frac{1}{2 \pi} \int_{0}^{2 \pi}\left|f^{(n)}\left(r e^{i \theta}\right)\right|^{\lambda} d \theta\right)^{1 / \lambda} \quad(f \in \widetilde{\mathcal{A}})
$$

is continuous on $\widetilde{\mathcal{A}}$. For $\lambda \geq 1$, by Minkowski's inequality it is also convex on $\widetilde{\mathcal{A}}$.

The extreme point theory for analytic functions was intensively investigated by Hallenbeck and MacGregor [9] (see also [3], [7], [8] and [16]).

Let $\Omega$ denote the class of $\omega \in \widetilde{\mathcal{A}}$ such that

$$
|\omega(z)| \leq|z| \quad(z \in \mathcal{U}) \text {. }
$$

We say that a function $f \in \widetilde{\mathcal{A}}$ is subordinate to a function $F \in \widetilde{\mathcal{A}}$, and write $f(z) \prec F(z)$ (or simply $f \prec F$ ), if and only if there exists a function $\omega \in \Omega$ such that

$$
f(z)=F(\omega(z)) \quad(z \in \mathcal{U}) .
$$

In particular, if $F$ is univalent in $\mathcal{U}$, we have the following equivalence:

$$
f(z) \prec F(z) \Leftrightarrow f(0)=F(0) \text { and } f(\mathcal{U}) \subset F(\mathcal{U}) .
$$

Also, we denote

$$
s(F):=\{f \in \widetilde{\mathcal{A}}: f \prec F\} .
$$

For functions $f, g \in \widetilde{\mathcal{A}}$ of the form

$$
f(z)=\sum_{n=0}^{\infty} a_{n} z^{n} \quad \text { and } \quad g(z)=\sum_{n=0}^{\infty} b_{n} z^{n},
$$


we denote by $f * g$ their Hadamard product (or convolution), defined by

$$
(f * g)(z)=\sum_{n=0}^{\infty} a_{n} b_{n} z^{n} \quad(z \in \mathcal{U}) .
$$

We denote by $\mathcal{A}$ the class of functions $f \in \widetilde{\mathcal{A}}$ of the form

$$
f(z)=z+\sum_{n=2}^{\infty} a_{n} z^{n} \quad(z \in \mathcal{U}) .
$$

Also, let $\mathcal{T}_{\eta}(\eta \in \mathbb{R})$ denote the class of functions $f \in \mathcal{A}$ of the form (5) for which

$$
\arg \left(a_{n}\right)=\pi+(1-n) \eta \quad(n=2,3, \ldots) .
$$

In particular, for $\eta=0$ we obtain the class $\mathcal{T}_{0}$ of functions with negative coefficients. Moreover, we define

$$
\mathcal{T}:=\bigcup_{\eta \in \mathbb{R}} \mathcal{T}_{\eta}
$$

The class $\mathcal{T}$ was introduced by Silverman [17] (see also [20]). It is called the class of functions with varying argument of coefficients.

Let $A, B$ be real parameters, $-1 \leq A<B \leq 1$, and let $\varphi, \phi \in \mathcal{A}$ be given functions of the form

$$
\varphi(z)=z+\sum_{n=2}^{\infty} \alpha_{n} z^{n}, \quad \phi(z)=z+\sum_{n=2}^{\infty} \beta_{n} z^{n} \quad(z \in \mathcal{U}),
$$

where the sequences $\left\{\alpha_{n}\right\},\left\{\beta_{n}\right\}$ are real and

$$
0 \leq \alpha_{n}<\beta_{n} \quad(n=2,3, \ldots) .
$$

Moreover, we assume

$$
d_{n}:=(1+B) \beta_{n}-(1+A) \alpha_{n} \quad(n=2,3, \ldots), \quad \liminf _{n \rightarrow \infty} \sqrt[n]{d_{n}} \geq 1 .
$$

We denote by $\mathcal{W}(\phi, \varphi ; A, B)$ the class of functions $f \in \mathcal{A}$ such that

$$
\frac{(\phi * f)(z)}{(\varphi * f)(z)} \prec \frac{1+A z}{1+B z},
$$

and we set

$$
\begin{aligned}
\mathcal{W}(\varphi ; A, B) & :=\mathcal{W}\left(z \varphi^{\prime}(z), \varphi(z) ; A, B\right), \\
\mathcal{W}(\varphi ; \alpha) & :=\mathcal{W}(\varphi ; 2 \alpha-1,1) \quad(0 \leq \alpha<1)
\end{aligned}
$$

In particular,

$$
\mathcal{S}^{*}(\alpha):=\mathcal{W}\left(\frac{z}{1-z} ; \alpha\right), \quad \mathcal{S}^{c}(\alpha):=\mathcal{W}\left(\frac{z}{(1-z)^{2}} ; \alpha\right)
$$

are the well-known classes of starlike functions of order $\alpha$ and convex functions of order $\alpha$, respectively. 
If we denote

$$
\mathcal{P}(A, B):=s\left(\frac{1+A z}{1+B z}\right)
$$

and

$$
\mathcal{P}(\alpha):=\mathcal{P}(2 \alpha-1,1), \quad \mathcal{P}:=\mathcal{P}(0) \quad(0 \leq \alpha<1),
$$

then we observe that

$$
f \in \mathcal{W}(\phi, \varphi ; A, B) \Leftrightarrow \frac{\phi * f}{\varphi * f} \in \mathcal{P}(A, B),
$$

and

$$
p \in \mathcal{P} \Leftrightarrow(1-\alpha) p(z)+\alpha \equiv q(z) \in \mathcal{P}(\alpha) .
$$

Finally, we define classes of functions with varying argument of coefficients related to the class $\mathcal{W}(\phi, \varphi ; A, B)$. Let us denote

$$
\begin{aligned}
\mathcal{T} \mathcal{W}(\phi, \varphi ; A, B) & :=\mathcal{T} \cap \mathcal{W}(\phi, \varphi ; A, B), \\
\mathcal{T} \mathcal{W}_{\eta}(\phi, \varphi ; A, B) & :=\mathcal{T}_{\eta} \cap \mathcal{W}(\phi, \varphi ; A, B) .
\end{aligned}
$$

The families $\mathcal{W}(\phi, \varphi ; A, B)$ and $\mathcal{T W}_{\eta}(\phi, \varphi ; A, B)$ unify various new and also well-known classes of analytic functions. We list a few of them in the last section.

The object of the present paper is to investigate extreme points of the classes $\mathcal{W}(\varphi ; A, B)$ and $\mathcal{T} \mathcal{W}_{\eta}(\phi, \varphi ; A, B)$. By using extreme point theory we obtain coefficient estimates and distortion theorems in these classes of functions. Some integral mean inequalities are also pointed out.

2. Extreme points. First we consider extreme points of $\mathcal{W}(\varphi ; \alpha)$. Using the Herglotz formula for Carathéodory functions and the relationship 12 we obtain

Lemma 1. A function $p$ belongs to the class $\mathcal{P}(\alpha)$ if and only if there is a probability measure $\mu$ on $\partial \mathcal{U}$ such that

$$
p(z)=\int_{|x|=1} \frac{1+(2 \alpha-1) x z}{1+x z} d \mu(x) \quad(z \in \mathcal{U}) .
$$

The correspondence between $\mathcal{P}$ and probability measures $\mu$ on $\partial \mathcal{U}$ given trough (13) is one-to-one.

Using Lemma 1 we get the following lemma.

Lemma 2. A function $f$ belongs to the class $\mathcal{S}^{*}(\alpha)$ if and only if there exists a probability measure $\mu$ on $\partial \mathcal{U}$ such that

$$
f(z)=z \exp \int_{|x|=1}\{-2(1-\alpha) \log (1+x z)\} d \mu(x) \quad(z \in \mathcal{U}) .
$$


The correspondence between $\mathcal{S}^{*}(\alpha)$ and probability measures $\mu$ on $\partial \mathcal{U}$ is oneto-one.

Let $f \in \mathcal{S}^{*}(\alpha)$. Then by Lemma 2 we have

$$
\frac{f(z)}{z}=\exp \int_{|x|=1}\{-2(1-\alpha) \log (1+x z)\} d \mu(x) \quad(z \in \mathcal{U}) .
$$

Since the function $k(z)=\log (1+z)$ is univalent and convex in $\mathcal{U}$, we have

$$
\int_{|x|=1} \log (1+x z) d \mu(x) \prec k(z),
$$

and consequently

$$
\frac{f(z)}{z}=\exp \{-2(1-\alpha) \log (1+\omega(z))\} \quad(z \in \mathcal{U})
$$

for some $\omega \in \Omega$. Hence by definition of subordination we have the following well-known result.

Lemma 3. If $f \in \mathcal{S}^{*}(\alpha)$, then

$$
\frac{f(z)}{z} \prec \frac{1}{(1+z)^{2(1-\alpha)}} .
$$

Lemma $4([9])$. Let

$$
F_{a}(z)=\frac{1}{(1+z)^{a}} \quad(z \in \mathcal{U}, a \geq 1) .
$$

A function $f \in \widetilde{\mathcal{A}}$ belongs to the class $H s\left(F_{a}\right)$ if and only if it can be represented by the formula

$$
f(z)=\int_{|x|=1} \frac{1}{(1+x z)^{a}} d \mu(x) \quad(z \in \mathcal{U}),
$$

where $\mu$ is a probability measure on $\partial \mathcal{U}$.

Theorem 1. Let $0 \leq \alpha \leq 1 / 2$. A function $f \in \mathcal{A}$ belongs to the class $H_{\mathcal{S}}{ }^{*}(\alpha)$ if and only if it can be represented by the formula

$$
f(z)=\int_{|x|=1} \frac{z}{(1+x z)^{2(1-\alpha)}} d \mu(x) \quad(z \in \mathcal{U}),
$$

where $\mu$ is a probability measure on $\partial \mathcal{U}$. Also,

$$
\operatorname{EHS}^{*}(\alpha)=\left\{\frac{z}{(1+x z)^{2(1-\alpha)}}:|x|=1\right\} .
$$

Proof. Let $\mathcal{G}$ be the class of functions represented by 15 . It is clear that $H \mathcal{G}=\mathcal{G}$. If $f \in H \mathcal{S}^{*}(\alpha)$, then according to Lemma 3 we have $f(z) / z \in$ 
$H s\left(F_{2(1-\alpha)}\right)$, where $F_{2(1-\alpha)}$ is defined by $(14)$. Thus, by Lemma 4 we have (15), i.e.

$$
H \mathcal{S}^{*}(\alpha) \subset H \mathcal{G}=\mathcal{G} \text {. }
$$

Since $E \mathcal{G} \subset \mathcal{S}^{*}(\alpha)$, we get $\mathcal{G} \subset H \mathcal{S}^{*}(\alpha)$, and therefore $\mathcal{G}=H \mathcal{S}^{*}(\alpha)$. Moreover, $E \mathcal{G}=E H^{*}(\alpha)$ and we obtain $(16)$.

REMARK 1 . We can represent the extreme points of the class $\mathcal{S}^{*}(\alpha)$ in the following form:

$$
\frac{z}{(1+x z)^{2(1-\alpha)}}=z+\sum_{n=2}^{\infty} \frac{(2-2 \alpha)_{n-1}}{(n-1) !}(-x)^{n-1} z^{n} \quad(z \in \mathcal{U}),
$$

where $(\lambda)_{n}$ is the Pochhammer symbol defined by

$$
(\lambda)_{n}= \begin{cases}1, & n=0 \\ \lambda(\lambda+1) \cdots(\lambda+n-1), & n \in \mathbb{N} .\end{cases}
$$

Theorem 2. Let $\left\{\alpha_{n}\right\}$ be defined by (8), $\alpha_{n}>0(n=2,3, \ldots)$, and

$$
\lim _{n \rightarrow \infty} \sqrt[n]{\alpha_{n}}=1, \quad 0 \leq \alpha \leq 1 / 2
$$

Then

$$
\operatorname{EH\mathcal {W}}(\varphi ; \alpha)=\left\{\varphi_{x}:|x|=1\right\}
$$

where

$$
\varphi_{x}(z):=z+\sum_{n=2}^{\infty} \frac{(2-2 \alpha)_{n-1}}{(n-1) ! \alpha_{n}}(-x)^{n-1} z^{n} \quad(z \in \mathcal{U},|x|=1) .
$$

Proof. Let

$$
\varphi^{\dagger}(z)=z+\sum_{n=2}^{\infty} \frac{1}{\alpha_{n}} z^{n} \quad(z \in \mathcal{U}) .
$$

Then the linear homeomorphism

$$
I(f)=\varphi^{\dagger} * f \quad(f \in \mathcal{A})
$$

maps $\mathcal{S}^{*}(\alpha)$ onto $\mathcal{W}(\varphi ; \alpha)$. Therefore, by (1) and Theorem 1 we obtain 18 .

Now, we consider extreme points of the class $\mathcal{T W}_{\eta}(\phi, \varphi ; A, B)$. First we mention a sufficient condition for a function to belong to $\mathcal{W}(\phi, \varphi ; A, B)$.

Theorem 3. Let $\left\{d_{n}\right\}$ be defined by (9) and $-1 \leq A<1,0 \leq B \leq 1$. If a function $f \in \mathcal{A}$ of the form (5) satisfies the condition

$$
\sum_{n=2}^{\infty} d_{n}\left|a_{n}\right| \leq B-A
$$

then $f \in \mathcal{W}(\phi, \varphi ; A, B)$. 
Proof. A function $f \in \mathcal{A}$ of the form (5) belongs to $\mathcal{W}(\phi, \varphi ; A, B)$ if and only if there exists $\omega \in \Omega$ such that

$$
\frac{(\phi * f)(z)}{(\varphi * f)(z)}=\frac{1+A \omega(z)}{1+B \omega(z)} \quad(z \in \mathcal{U})
$$

or equivalently

$$
\left|\frac{(\phi * f)(z)-(\varphi * f)(z)}{B(\phi * f)(z)-A(\varphi * f)(z)}\right|<1 \quad(z \in \mathcal{U}) .
$$

Thus, it is sufficient to prove that

$$
\left|\frac{(\phi * f)(z)-(\varphi * f)(z)}{z}\right|-\left|\frac{B(\phi * f)(z)-A(\varphi * f)(z)}{z}\right|<0 \quad(z \in \mathcal{U}) .
$$

Indeed, letting $|z|=r(0<r<1)$ we have

$$
\begin{aligned}
& \left|\frac{(\phi * f)(z)-(\varphi * f)(z)}{z}\right|-\left|\frac{B(\phi * f)(z)-A(\varphi * f)(z)}{z}\right| \\
& \quad=\left|\sum_{n=2}^{\infty}\left(\beta_{n}-\alpha_{n}\right) a_{n} z^{n-1}\right|-\left|(B-A)-\sum_{n=2}^{\infty}\left(B \beta_{n}-A \alpha_{n}\right) a_{n} z^{n-1}\right| \\
& \quad \leq \sum_{n=2}^{\infty}\left(\beta_{n}-\alpha_{n}\right)\left|a_{n}\right| r^{n-1}-(B-A)+\sum_{n=2}^{\infty}\left(B \beta_{n}-A \alpha_{n}\right)\left|a_{n}\right| r^{n-1} \\
& \quad=\sum_{n=2}^{\infty} d_{n}\left|a_{n}\right| r^{n-1}-(B-A)<0 .
\end{aligned}
$$

TheOREM 4. Let $f \in \mathcal{A}$ be a function of the form (5), satisfying (6). Then $f \in \mathcal{T W}_{\eta}(\phi, \varphi ; A, B)$ if and only if the condition (20) holds true.

Proof. In view of Theorem 3 we need only show that if the function $f$ is in $\mathcal{T W}_{\eta}(\phi, \varphi ; A, B)$ then it satisfies $(20)$. So assume $f \in \mathcal{T W}_{\eta}(\phi, \varphi ; A, B)$. Then, by (5) and (21) we have

$$
\left|\frac{\sum_{n=2}^{\infty}\left(\beta_{n}-\alpha_{n}\right) a_{n} z^{n-1}}{B-A-\sum_{n=2}^{\infty}\left(B \beta_{n}-A \alpha_{n}\right) a_{n} z^{n-1}}\right|<1 \quad(z \in \mathcal{U}) .
$$

Therefore, putting $z=r e^{i \eta}(0 \leq r<1)$ and applying (6) we obtain

$$
\frac{\sum_{n=2}^{\infty}\left(\beta_{n}-\alpha_{n}\right)\left|a_{n}\right| r^{n-1}}{B-A-\sum_{n=2}^{\infty}\left(B \beta_{n}-A \alpha_{n}\right)\left|a_{n}\right| r^{n-1}}<1 .
$$

It is clear that the denominator above cannot vanish for $r \in[0,1)$. Moreover, it is positive for $r=0$, and hence for $r \in[0,1)$. Thus, by (22) we have

$$
\sum_{n=2}^{\infty}\left[(1+B) \beta_{n}-(1+A) \alpha_{n}\right]\left|a_{n}\right| r^{n-1}<B-A,
$$

which, upon letting $r \rightarrow 1^{-}$, readily yields 20 . 
Since the condition 20 is independent of $\eta$, Theorem 4 yields the following theorem.

TheOREm 5. Let $f \in \mathcal{A}$ be a function of the form (5), satisfying (6). Then $f \in \mathcal{T} \mathcal{W}(\phi, \varphi ; A, B)$ if and only if the condition (20) holds true.

Since $\widetilde{\mathcal{A}}$ is a complete metric space, Montel's theorem (see [13]) implies the following lemma.

LEMma 5. A class $\mathcal{F}$ contained in $\widetilde{\mathcal{A}}$ is compact if and only if $\mathcal{F}$ is closed and locally uniformly bounded.

Theorem 6. The class $\mathcal{T W}_{\eta}(\phi, \varphi ; A, B)$ is convex and compact.

Proof. Let $f, g \in \mathcal{T} \mathcal{W}_{\eta}(\phi, \varphi ; A, B), 0 \leq \gamma \leq 1$. Since

$$
\begin{aligned}
\gamma f(z)+(1-\gamma) g(z) & =\gamma\left(z+\sum_{n=2}^{\infty} a_{n} z^{n}\right)+(1-\gamma)\left(z+\sum_{n=2}^{\infty} b_{n} z^{n}\right) \\
& =z+\sum_{n=2}^{\infty}\left(\gamma a_{n}+(1-\gamma) b_{n}\right) z^{n},
\end{aligned}
$$

by Theorem 4 we have

$$
\begin{aligned}
\sum_{n=2}^{\infty} d_{n}\left|\gamma a_{n}+(1-\gamma) b_{n}\right| & \leq \gamma \sum_{n=2}^{\infty} d_{n}\left|a_{n}\right|+(1-\gamma) \sum_{n=2}^{\infty} d_{n}\left|b_{n}\right| \\
& \leq \gamma(B-A)+(1-\gamma)(B-A)=B-A,
\end{aligned}
$$

and consequently $h=\gamma f+(1-\gamma) g \in \mathcal{T W}_{\eta}(\phi, \varphi ; A, B)$. Hence this class is convex. Furthermore, for $f \in \mathcal{T W}_{\eta}(\phi, \varphi ; A, B),|z| \leq r, 0<r<1$, we have

$$
|f(z)| \leq r+\sum_{n=2}^{\infty} d_{n} a_{n} \frac{r^{n}}{d_{n}} \leq r+(B-A) \sum_{n=2}^{\infty} \frac{r^{n}}{d_{n}} .
$$

By (9) we have

$$
\limsup _{n \rightarrow \infty}\left(r^{n} d_{n}^{-1}\right)^{1 / n}=\frac{r}{\liminf _{n \rightarrow \infty} \sqrt[n]{d_{n}}} \leq r<1 .
$$

Thus, the power series $\sum_{n=2}^{\infty} r^{n} d_{n}^{-1}$ converges and by 23 we conclude that the class $\mathcal{T W}_{\eta}(\phi, \varphi ; A, B)$ is locally uniformly bounded. By Lemma 5, we only need to show that it is closed. Let $f_{m} \in \mathcal{T}_{\mathcal{W}}(\phi, \varphi ; A, B)(m \in \mathbb{N})$ and $f_{m} \rightarrow f$. Suppose that

$$
f_{m}(z)=z+\sum_{n=2}^{\infty} a_{n, m} z^{n} \quad(m \in \mathbb{N}, z \in \mathcal{U})
$$

and $f$ is given by (5). Using Theorem 1 we have

$$
\sum_{n=2}^{\infty} d_{n}\left|a_{n, m}\right| \leq B-A \quad(m \in \mathbb{N}) .
$$


Since $f_{m} \rightarrow f$, we conclude that $a_{n, m} \rightarrow a_{n}$ as $m \rightarrow \infty(n \in \mathbb{N})$. This gives the condition $(20)$, and so $f \in \mathcal{T W}_{\eta}(\phi, \varphi ; A, B)$, which completes the proof.

THEOREM 7.

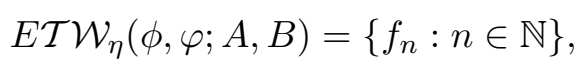

where $f_{1}(z)=z$ and

$$
f_{n}(z)=f_{n, \eta}(z)=z-\frac{B-A}{d_{n}} e^{i(1-n) \eta} z^{n} \quad(n=2,3, \ldots, z \in \mathcal{U}) .
$$

Proof. By using (20) we easily verify that all functions of the form 25) are extreme points of $\mathcal{T} \mathcal{W}_{\eta}(\phi, \varphi ; A, B)$. Now, suppose $f \in \operatorname{E\mathcal {T}}_{\eta}(\phi, \varphi ; A, B)$ and $f$ is not of the form $(25)$. If

$$
f(z)=z-\gamma \frac{B-A}{d_{n}} z^{n} \quad(0<\gamma<1, n=2,3, \ldots, z \in \mathcal{U}),
$$

then

$$
f(z)=(1-\gamma) f_{1}(z)+\gamma f_{n}(z)(z \in \mathcal{U}),
$$

and so $f$ is not an extreme point of $\mathcal{T W}_{\eta}(\phi, \varphi ; A, B)$. In the opposite case there exist $m, l \in \mathbb{N}, m \neq l$, so that the coefficients $a_{m}$ and $a_{l}$ do not vanish in the power series (5). Putting

$$
\begin{aligned}
g(z) & =f(z)-a_{l} z^{l}+\frac{a_{l}}{d_{m}} z^{m}, \\
h(z) & =f(z)-a_{m} z^{m}+\frac{a_{m}}{d_{l}} z^{l}, \\
\gamma & =\frac{d_{m} a_{m}}{d_{m} a_{m}+d_{l} a_{l}},
\end{aligned}
$$

we have

$$
g, h \in \mathcal{T W}_{\eta}(\phi, \varphi ; A, B), \quad g \neq h, \quad 0<\gamma<1 \quad \text { and } \quad f=\gamma g+(1-\gamma) h .
$$

It follows that $f \notin E \mathcal{T W}_{\eta}(\phi, \varphi ; A, B)$, and the proof is complete.

3. Applications. The Krein-Milman theorem (see [10] and [15]) is fundamental in the theory of extreme points.

Lemma 6 (Krein-Milman theorem). If $\mathcal{F}$ is a compact convex subclass of $\mathcal{A}$, then $H E \mathcal{F}=\mathcal{F}$.

In particular, the Krein-Milman implies the following important result.

Lemma 7 ([9]). Let $\mathcal{F}$ be a compact convex subclass of the class $\widetilde{\mathcal{A}}$ and $J: \widetilde{\mathcal{A}} \rightarrow \mathbb{R}$ be a continuous and convex functional on $\mathcal{F}$. Then

$$
\max \{\mathcal{J}(f): f \in H \mathcal{F}\}=\max \{\mathcal{J}(f): f \in \mathcal{F}\}=\max \{\mathcal{J}(f): f \in E H \mathcal{F}\} .
$$


Lemma 8 ([11]). Let $f, g \in \widetilde{\mathcal{A}}$. If $f \prec g$, then

$$
\int_{0}^{2 \pi}\left|f\left(r e^{i \theta}\right)\right|^{\lambda} d \theta \leq \int_{0}^{2 \pi}\left|g\left(r e^{i \theta}\right)\right|^{\lambda} d \theta \quad(0<r<1, \lambda>0) .
$$

By using (2) and Theorems 6 and 7, the Krein-Milman theorem gives the following corollary.

Corollary 1.

$$
\mathcal{T W}_{\eta}(\phi, \varphi ; A, B)=\left\{\sum_{n=1}^{\infty} \gamma_{n} f_{n, \eta}: \sum_{n=2-1}^{\infty} \gamma_{n}=1, \gamma_{n} \geq 0(n \in \mathbb{N})\right\},
$$

where $f_{1}(z)=z$ and $f_{n, \eta}$ are defined by (25).

Moreover, by Theorem 2 we obtain

Corollary 2. Let $\varphi_{x}$ be defined by (19), and suppose (17) holds. Then the class $H \mathcal{W}(\varphi ; \alpha)$ contains all functions $f \in \mathcal{A}$ represented by the formula

$$
f(z)=\int_{|x|=1} \varphi_{x}(z) d \mu(x) \quad(z \in \mathcal{U})
$$

where $\mu$ is a probability measure on $\partial \mathcal{U}$.

Using the extremal points of the classes $\mathcal{W}(\varphi ; \alpha)$ and $\mathcal{T W}_{\eta}(\phi, \varphi ; A, B)$ we obtain some results listed below. Combining (3) with Lemma 7 yields the following three corollaries:

Corollary 3. Let $\varphi_{x}$ be defined by (19), and suppose (17) holds. If a function $f$ of the form (5) belongs to the class $\mathcal{W}(\varphi ; \alpha)$, then

$$
\left|a_{n}\right| \leq \frac{(2-2 \alpha)_{n-1}}{(n-1) ! \alpha_{n}} \quad(n=2,3, \ldots) .
$$

The result is sharp. The functions $\varphi_{x}$ are the extremal functions.

COROllary 4. If a function $f$ of the form (5) belongs to the class $\mathcal{T W}_{\eta}(\phi, \varphi ; A, B)$, then

$$
\left|a_{n}\right| \leq \frac{B-A}{d_{n}} \quad(n=2,3, \ldots),
$$

where $d_{n}$ is defined by (9). The result is sharp. The functions $f_{n, \eta}$ of the form (25) are the extremal functions.

Corollary 5. Let $\varphi_{x}$ be defined by (19), and suppose (17) holds. If $f \in \mathcal{W}(\varphi ; \alpha)$, then

$$
\min _{|z|=r} \varphi_{1}^{(k)}(z) \leq\left|f^{(k)}(z)\right| \leq \varphi_{1}^{(k)}(r) \quad(k=0,1, \ldots,|z|=r<1) .
$$

The result is sharp. The functions $\varphi_{x}$ are the extremal functions. 
For the extreme points $f_{n, \eta}$ of the form $(25)$ we have

$$
\begin{aligned}
& f_{n, \eta}^{\prime}(z)=1-\frac{(B-A) n}{d_{n}} e^{i(1-n) \eta} z^{n-1}, \\
& f_{n, \eta}^{(k)}(z)=-\frac{(B-A) n !}{(n-k) ! d_{n}} e^{i(1-n) \eta} z^{n-k} \quad(k=2, \ldots, n), \\
& f_{n, \eta}^{(k)}(z)=0 \quad(k=n+1, n+2, \ldots) .
\end{aligned}
$$

Let $k \in \mathbb{N}_{0}=\mathbb{N} \cup\{0\}, 0<r<1$, and define the sequence $\left\{\delta_{n}^{(k)}\right\}$ by

$$
\delta_{n}^{(k)}=\frac{(B-A) n !}{(n-k) ! d_{n}} r^{n-k} \quad(n \geq \max \{k, 2\}) .
$$

Applying (24) we obtain

$$
\limsup _{n \rightarrow \infty} \delta_{n}^{(k)}=0 \quad\left(k \in \mathbb{N}_{0}\right) .
$$

Thus, there exist $n_{k} \in \mathbb{N}\left(k \in \mathbb{N}_{0}\right)$ such that

$$
\delta_{n_{k}}^{(k)}=\max \left\{\delta_{n}^{(k)}: n \geq \max \{k, 2\}\right\} \quad\left(k \in \mathbb{N}_{0}\right) .
$$

Therefore, by Lemma 7 we have the following corollary.

Corollary 6. If $f \in \mathcal{T W}_{\eta}(\phi, \varphi ; A, B)$ and $|z|=r<1$, then

$$
\begin{aligned}
r-\frac{B-A}{d_{n_{0}}} r^{n_{0}} \leq|f(z)| & \leq r+\frac{B-A}{d_{n_{0}}} r^{n_{0}}, \\
1-\frac{(B-A) n_{1}}{d_{n_{1}}} r^{n_{1}} \leq\left|f^{\prime}(z)\right| & \leq 1+\frac{(B-A) n_{1}}{d_{n_{1}}} r^{n_{1}}, \\
\left|f^{(k)}(z)\right| & \leq \frac{(B-A)\left(n_{k}\right) !}{\left(n_{k}-k\right) ! d_{n_{k}}} r^{n_{k}-k} \quad(k \geq 2),
\end{aligned}
$$

where $n_{k}$ is defined by (29). The result is sharp. The functions $f_{n_{k}, \eta}$ of the form (25) are the extremal functions.

From Corollary 6 we have the following corollary.

Corollary 7. Let $f \in \mathcal{T} \mathcal{W}_{\eta}(\phi, \varphi ; A, B),|z|=r<1$, and $k \in \mathbb{N}_{0}$. If the sequence $\left\{\delta_{n}^{(k)}\right\}$ defined by 28) is nonincreasing with respect to $n$, then

$$
\begin{aligned}
r-\frac{B-A}{d_{2}} r^{2} \leq|f(z)| & \leq r+\frac{B-A}{d_{2}} r^{2} \quad(k=0), \\
1-\frac{2(B-A)}{d_{2}} r^{2} \leq\left|f^{\prime}(z)\right| & \leq 1+\frac{2(B-A)}{d_{2}} r^{2} \quad(k=1), \\
\left|f^{(k)}(z)\right| & \leq \frac{(B-A) k !}{d_{k}} \quad(k=2,3, \ldots),
\end{aligned}
$$

where $n_{k}$ is defined by (29). The result is sharp. The functions $f_{n_{k}, \eta}$ of the form (25) are the extremal functions. 
Now, we consider some integral mean inequalities. By (4), Lemma 7 yields the following corollary.

Corollary 8. Let $\varphi_{x}$ be defined by (19), and suppose (17) holds. If $f \in \mathcal{W}(\varphi ; \alpha)$, then

$$
\int_{0}^{2 \pi}\left|f^{(k)}\left(r e^{i \theta}\right)\right|^{\lambda} d \theta \leq \int_{0}^{2 \pi}\left|\varphi_{1}^{(k)}\left(r e^{i \theta}\right)\right|^{\lambda} d \theta \quad(0<r<1, \lambda \geq 1) .
$$

Corollary 9. Let $0<r<1, \lambda \geq 1, k \in \mathbb{N}_{0}$ and assume that the sequence $\left\{\delta_{n}^{(k)}\right\}$ defined by 28 is nonincreasing with respect to $n$. If $f \in$ $\mathcal{T W}_{\eta}(\phi, \varphi ; A, B)$, then

$$
\begin{array}{ll}
\frac{1}{2 \pi} \int_{0}^{2 \pi}\left|f^{(k)}\left(r e^{i \theta}\right)\right|^{\lambda} d \theta \leq \frac{1}{2 \pi} \int_{0}^{2 \pi}\left|f_{2, \eta}^{(k)}\left(r e^{i \theta}\right)\right|^{\lambda} d \theta & (k=0,1), \\
\frac{1}{2 \pi} \int_{0}^{2 \pi}\left|f^{(k)}\left(r e^{i \theta}\right)\right|^{\lambda} d \theta \leq \frac{1}{2 \pi} \int_{0}^{2 \pi}\left|f_{k, \eta}^{(k)}\left(r e^{i \theta}\right)\right|^{\lambda} d \theta \quad(k=2,3, \ldots),
\end{array}
$$

where $f_{k, \eta}$ are the functions defined by (25).

Proof. Since

$$
\frac{f_{n, \eta}}{z} \prec \frac{f_{2, \eta}}{z} \quad \text { and } \quad f_{n, \eta}^{\prime} \prec f_{2, \eta}^{\prime} \quad(n \in \mathbb{N}),
$$

using (7) and Lemma 8 we have

$$
\max \left\{\int_{0}^{2 \pi}\left|f_{n, \eta}^{(k)}\left(r e^{i \theta}\right)\right|^{\lambda} d \theta: n \in \mathbb{N}\right\}=\int_{0}^{2 \pi}\left|f_{2, \eta}^{(k)}\left(r e^{i \theta}\right)\right|^{\lambda} d \theta \quad(k=0,1) .
$$

Thus, Lemma 7 yields (36). The inequality 37 is an immediate consequence of 35 and (27).

Making use of (7) and Corollaries 4, 6, 7 and 9, we get the corollaries listed below.

COROLlary 10. If a function $f$ of the form (5) belongs to the class $\mathcal{T W}(\phi, \varphi ; A, B)$, then the coefficient estimates (26) hold true. The result is sharp. The functions $f_{n, \eta}$ of the form $(25)(\eta \in \mathbb{R})$ are the extremal functions.

Corollary 11. If $f \in \mathcal{T} \mathcal{W}(\phi, \varphi ; A, B)$ and $|z|=r<1$, then the bounds (30)-32 hold true. The results are sharp. The functions $f_{n_{k}, \eta}(\eta \in \mathbb{R})$ of the form (25) are the extremal functions.

Corollary 12. Let $f \in \mathcal{T} \mathcal{W}(\phi, \varphi ; A, B),|z|=r<1$, and $k \in \mathbb{N}_{0}$. If the sequence $\left\{b_{n}^{(k)}\right\}$ defined by 28 is nonincreasing with respect to $n$, then the inequalities (33)-(35) hold true. The result is sharp. The functions $f_{n, \eta}$ $(\eta \in \mathbb{R})$ of the form (25) are the extremal functions. 
Corollary 13. Let $0<r<1, \lambda \geq 1, k \in \mathbb{N}_{0}$ and assume that the sequence $\left\{\delta_{n}^{(k)}\right\}$ defined by 28 is nonincreasing with respect to $n$. If $f \in$ $\mathcal{T} \mathcal{W}(\phi, \varphi ; A, B)$, then the inequalities (36) and (37) hold true.

4. Concluding remarks. We conclude this paper by observing that, in view of the subordination relation (10), choosing the functions $\phi$ and $\varphi$ appropriately, we can consider new and also well-known classes of functions. In particular, the class

$$
\mathcal{W}_{n}(\varphi ; A, B):=\mathcal{W}\left(z \varphi^{\prime}(z), \sum_{k=0}^{n-1} \varphi\left(x^{k} z\right) ; A, B\right),
$$

where $n \in \mathbb{N}, x^{n}=1$, consists of the functions $f \in \mathcal{A}$ such that

$$
\frac{z(\varphi * f)^{\prime}(z)}{\sum_{k=0}^{n-1}(\varphi * f)\left(x^{k} z\right)} \prec \frac{1+A z}{1+B z} .
$$

It is related to the class of starlike functions with respect to $n$-symmetric points. Moreover, for $n=1$, we obtain the class

$$
\mathcal{W}(\varphi ; A, B)=\mathcal{W}_{1}(\varphi ; A, B)
$$

defined by the following condition:

$$
\frac{z(\varphi * f)^{\prime}(z)}{(\varphi * f)(z)} \prec \frac{1+A z}{1+B z} .
$$

This class is related to the class of starlike functions.

Let $\lambda$ be a convex parameter. A function $f \in \mathcal{A}$ belongs to the class

$$
\mathcal{V}_{\lambda}(\varphi ; A, B):=\mathcal{W}\left(\lambda \frac{\varphi(z)}{z}+(1-\lambda) \varphi^{\prime}(z), z ; A, B\right)
$$

if it satisfies the following condition:

$$
\lambda \frac{(\varphi * f)(z)}{z}+(1-\lambda)(\varphi * f)^{\prime}(z) \prec \frac{1+A z}{1+B z} .
$$

Likewise, a function $f \in \mathcal{A}$ belongs to the class

$$
\mathcal{U}_{\lambda}(\varphi ; A, B):=\mathcal{W}\left(\lambda \frac{\varphi(z)}{z}+(1-\lambda) \varphi^{\prime}(z) ; A, B\right)
$$

if it satisfies the following condition:

$$
\frac{z(\varphi * f)^{\prime}(z)+(1-\lambda) z^{2}(\varphi * f)^{\prime \prime}(z)}{\lambda(\varphi * f)(z)+(1-\lambda) z(\varphi * f)^{\prime}(z)} \prec \frac{1+A z}{1+B z} .
$$

The classes $\mathcal{W}_{n}^{p}(\varphi ; A, B), \mathcal{U}_{\lambda}(\varphi ; A, B)$ and $\mathcal{V}_{\lambda}(\varphi ; A, B)$ generalize well-known important classes, which were investigated in earlier works (see for example [1, 4, 14, 18, 19, 12]). Most of these classes were defined by using linear operators and special functions. 
If we apply the results of this paper to the classes discussed above, we can get several additional new results. Some of these classes were obtained in earlier works (see for example [2, 5, 6, 21]).

\section{References}

[1] M. K. Aouf, H. Silverman and H. M. Srivastava, Some families of linear operators associated with certain subclasses of multivalent functions, Comput. Math. Appl. 55 (2008), 535-549.

[2] M. K. Aouf and H. M. Srivastava, Some families of starlike functions with negative coefficients, J. Math. Anal. Appl. 203 (1996), 762-790.

[3] J. E. Brown, Applications of extreme points to distortion estimates, Complex Var. Theory Appl. 47 (2002), 229-237.

[4] N. E. Cho and H. M. Srivastava, Argument estimates of certain analytic functions defined by a class of multiplier transformations, Math. Comput. Modelling 37 (2003), 39-49.

[5] J. Dziok, On the convex combination of the Dziok-Srivastava operator, Appl. Math. Comput. 188 (2007), 1214-1220.

[6] J. Dziok and H. M. Srivastava, Classes of analytic functions associated with the generalized hypergeometric function, ibid. 103 (1999), 1-13.

[7] D. J. Hallenbeck and K. T. Hallenbeck, Classes of analytic functions subordinate to convex functions and extreme points, J. Math. Anal. Appl. 282 (2003), 792-800.

[8] - , - Extreme points and support points of subordination families, ibid. 251 (2000), $157-166$.

[9] D. J. Hallenbeck and T. H. MacGregor, Linear Problems and Convexity Techniques in Geometric Function Theory, Pitman Adv. Publishing Program, Pitman, Boston, 1984.

[10] M. Krein and D. Milman, On extreme points of regular convex sets, Studia Math. 9 (1940), 133-138.

[11] J. E. Littlewood, On inequalities in the theory of functions, Proc. London Math. Soc. 23 (1925), 481-519.

[12] J.-L. Liu and H. M. Srivastava, Certain properties of the Dziok-Srivastava operator, Appl. Math. Comput. 159 (2004), 485-493.

[13] P. Montel, Sur les familles de fonctions analytiques qui admettent des valeurs exceptionnelles dans un domaine, Ann. Sci. École Norm. Sup. 23 (1912), 487-535.

[14] J. Patel, A. K. Mishra and H. M. Srivastava, Classes of multivalent analytic functions involving the Dziok-Srivastava operator, Comput. Math. Appl. 54 (2007), 599616.

[15] H. L. Royden, Real Analysis, Prentice-Hall, Englewood Cliffs, NJ, 1988.

[16] N. Samaris, The extreme points of some convex subsets of holomorphic functions, Math. Japon. 38 (1993), 735-737.

[17] H. Silverman, Univalent functions with varying arguments, Houston J. Math. 7 (1981), 283-287.

[18] J. Sokół, On a class of analytic multivalent functions, Appl. Math. Comput. 203 (2008), 210-216.

[19] - , On some applications of the Dziok-Srivastava operator, ibid. 201 (2008), 774780 . 
[20] H. M. Srivastava and S. Owa, Certain classes of analytic functions with varying arguments, J. Math. Anal. Appl. 136 (1988), 217-228.

[21] J. Stankiewicz and L. Trojnar-Spelina, Some operator properties of the special classes of functions with negative coefficients, Folia Sci. Univ. Techn. Resoviensis 127 (1994), 63-72.

Jacek Dziok

Institute of Mathematics

University of Rzeszów

35-310 Rzeszów, Poland

E-mail: jdziok@univ.rzeszow.pl

Received 8.5.2009

and in final form 18.12.2009 
